\title{
Coulomb Correlation and Information Entropies in Confined Helium-Like Atoms
}

\author{
Wallas Santos Nascimento ${ }^{\mathrm{a}, 1}$, Marcos Melo de Almeida ${ }^{\mathrm{b}, 1}$, Frederico \\ Vasconcellos Prudente ${ }^{c, 1}$ \\ ${ }^{1}$ Instituto de Física, Universidade Federal da Bahia, 40170-115, Salvador, Bahia, Brazil.
}

Version accepted for publication in Eur. Phys. J. D (2021). DOI: 10.1140/epjd/s10053-021-00177-6

\begin{abstract}
The present work studies aspects of the electronic correlation in confined $\mathrm{H}^{-}, \mathrm{He}$ and $\mathrm{Li}^{+}$atoms in their ground states using the informational entropies. In this way, different variational wavefunctions are employed in order of better take account of Coulomb correlation. The obtained values for the $S_{r}, S_{p}$ and $S_{t}$ entropies are sensitive in relation to Coulomb correlation effects. In the strong confinement regime, the effects of the Coulomb correlation are negligible and the employment of the models of independent particle and two non-interacting electrons confined by a impenetrable spherical cage gains importance in this regime. Lastly, energy values are obtained in good agreement with the results available in the literature.
\end{abstract}

Keywords Information entropies · Coulomb correlation · Confined quantum systems · Quantum information theory · Hylleraas coordinates.

\section{Introduction}

The problem of many bodies is best characterized when the constituent particles of the system interact simultaneously, that is, the particles have a correlated behavior. Specifically, the electronic interactions in atoms or molecules globally generate so-called correlation effects [1,2].

The phenomenon of electron correlation rises when working with models that go beyond the approximation of the independent particle model, being established by means of two essential origins. The first one is denominated Fermi correlation and results from the hypothesis of indistinguishability of the fermionic particles, producing an exchange force between them. The second one is known as Coulomb correlation based on the Coulomb repulsion between the electrons [3, 4].

Usually, the electronic correlation is studied as a guide in an energetic analysis of the systems. In this sense, the socalled correlation energy [5, 6], defined as the difference between the exact and limit Hartree-Fock energies, has received significant attention. The correlation energy, although small related to total energy, is very relevant in determination of chemical bonding energies. Futhermore, other energy analysis procedures can be defined [7, 8].

A mathematical theory of communication or information theory arose with report of Claude Shannon in 1948. A fundamental concept in this field is the information or Shannon entropy. This quantity is employed to measure the amount of information generated by a information source in the process of choosing a message from a possible group of them $[9,10]$. Information entropy is successfully used in the study of the most varied phenomena in diverse fields of expertise $[11,12]$.

From the connection between information and quantum theories, it emerges the informational entropies in position, $S_{r}$, and in momentum, $S_{p}$, spaces, and the entropy sum $S_{t}$ by adding $S_{r}$ and $S_{p}$. The study of quantum systems in information theory context already has a series of published works, for instance, analyses involving the one-dimensional systems [13-17] and confined hydrogen atom [18-23]. Studies on the free or confined helium-like atoms also have received attention in the informational context [24-33].

Spatially confined quantum systems are not a recent problem in physics and have their initial studies produced in the early 1930s [34-36]. Quantum mechanical properties of confined systems are influenced by properties of confinement cage, for instance, the energy spectra and the polarizability experience significant changes [37, 38]. With the increment of computational processing capacity and development of modern techniques for the effective confinement, this topic remains to be of interest [39-50]. In previous researches, we use the entropy sum to investigate the regions of the

\footnotetext{
ae-mail: wallassantos@gmail.com

be-mail: marcosma@ufba.br

ce-mail: prudente@ufba.br
} 
confined harmonic oscillator [51] and confined hydrogenic-like atoms [21] where the confinement effects has major impact.

Despite of the studies using energy analysis to measure electronic correlation, studies based on informational entropies are found in literature [52-57]. The investigation in free quantum systems, i.e., non spatially confined, shows promising results. For the lithium [58] and beryllium [59] atoms, and their ions, the $S_{t}$ values increase and the $S_{r}$ and $S_{p}$ values increase and decrease, respectively, using configuration interaction singles and doubles excitations instead of Hartree-Fock wavefunctions [60].

The goal of this work is to discuss the Coulomb correlation effects on the systems by means of their wavefunctions, employing informational entropies. The systems of interest are spatially confined atoms containing two electrons, namely: negative hydrogen ion $\left(\mathrm{H}^{-}\right)$, helium $(\mathrm{He})$ and ionized lithium $\left(\mathrm{Li}^{+}\right)$. This paper is organized as follows: in sect. 2 we present the informational concepts employed and we analyze the physical systems of interest together with the trial wavefunctions, in sect. 3 we discuss the obtained results and, finally, in sect. 4 we summarize the central aspects of our investigation. In Appendix A we present the $2 e^{-}$system.

\section{Theoretical background}

In this section, we present the concepts and methods applied in our research. In subsect. 2.1 we define the informational quantities $S_{r}, S_{p}$ and $S_{t}$. In subsect. 2.2 we examine the confined helium-like atoms, as being the physical system of interest in this work. Furthermore, we define the three trial wavefunctions that we use to describe the confined $\mathrm{H}^{-}, \mathrm{He}$ and $\mathrm{Li}^{+}$atoms in their ground energy states in order of better accuracy in the Coulomb correlation description.

\subsection{Information entropies}

The expression, originally introduced by Shannon, employed to measure the amount of information generated by a continuous information source is [10]

$$
S(p(\alpha))=-\int_{-\infty}^{\infty} d \alpha p(\alpha) \log _{2} p(\alpha)
$$

where $p(\alpha)$ is a probability density in function of continuous $\alpha$ variable, constrained by normalization condition, $\int_{-\infty}^{\infty} d \alpha p(\alpha)=1$, and non-negative condition, $p(\alpha) \geq 0, \forall \alpha$. Note that in the original Shannon's work the entropy values for continuous distributions may be negative (see pg. 631 in Ref. [10]).

Adopting on the expression (1) the two-electron probability densities in position and momentum spaces, respectively $\rho\left(\mathbf{r}_{1}, \mathbf{r}_{2}\right)$ and $\gamma\left(\mathbf{p}_{1}, \mathbf{p}_{2}\right)$, arising from quantum theory, and using the fundamental physical constants Bohr radius, $a_{0}$, and reduced Planck constant, $\hbar$, we have the information entropies in position and momentum spaces, respectively, defined as [21]

$$
S_{r}=-\iint d \mathbf{r}_{1} d \mathbf{r}_{2} \rho\left(\mathbf{r}_{1}, \mathbf{r}_{2}\right) \ln \left(a_{0}^{6} \rho\left(\mathbf{r}_{1}, \mathbf{r}_{2}\right)\right)
$$

and

$$
S_{p}=-\iint d \mathbf{p}_{1} d \mathbf{p}_{2} \gamma\left(\mathbf{p}_{1}, \mathbf{p}_{2}\right) \ln \left(\left(\frac{\hbar}{a_{0}}\right)^{6} \gamma\left(\mathbf{p}_{1}, \mathbf{p}_{2}\right)\right) .
$$

The $\rho\left(\mathbf{r}_{1}, \mathbf{r}_{2}\right)$ and $\gamma\left(\mathbf{p}_{1}, \mathbf{p}_{2}\right)$ probability densities are normalized to unity and are written in terms of two-electron wavefunctions, $\rho\left(\mathbf{r}_{1}, \mathbf{r}_{2}\right)=\left|\psi\left(\mathbf{r}_{1}, \mathbf{r}_{2}\right)\right|^{2}$ and $\gamma\left(\mathbf{p}_{1}, \mathbf{p}_{2}\right)=\left|\widetilde{\psi}\left(\mathbf{p}_{1}, \mathbf{p}_{2}\right)\right|^{2}$. The momentum space wavefunction $\widetilde{\psi}\left(\mathbf{p}_{1}, \mathbf{p}_{2}\right)$ is obtained by Fourier transform in the position space of wavefunction $\psi\left(\mathbf{r}_{1}, \mathbf{r}_{2}\right)$.

The $S_{t}$ quantity is obtained from entropy sum, $S_{r}+S_{p}$, as defined below. From the entropy sum we can still derive the entropy uncertainty relation that, written in terms of $\hbar$, is given by [21]

$$
\begin{aligned}
S_{t} & =S_{r}+S_{p} \\
& =-\int \cdots \int d \mathbf{r}_{1} d \mathbf{r}_{2} d \mathbf{p}_{1} d \mathbf{p}_{2} \rho\left(\mathbf{r}_{1}, \mathbf{r}_{2}\right) \gamma\left(\mathbf{p}_{1}, \mathbf{p}_{2}\right) \ln \left(\hbar^{6} \rho\left(\mathbf{r}_{1}, \mathbf{r}_{2}\right) \gamma\left(\mathbf{p}_{1}, \mathbf{p}_{2}\right)\right) \\
& \geq 6(1+\ln \pi) .
\end{aligned}
$$

From this entropy uncertainty relation we can obtain the Kennard's uncertainty relation [61]. The Eqs. (2), (3) and (4) are dimensionally adequate and independent of the employed unit system.

The $S_{r}$ and $S_{p}$ entropies are a type of dispersion or spread measures that are calculated without taking into account reference points in the probability distributions as Kennard's uncertainty relation (see, for example, Ref. [14] and references therein). In this sense, the entropic quantities are measures of uncertainty, localization or delocalization, of the wavefunction in the space $[14,62]$. 


\subsection{Systems of interest}

The confined two-electrons (or the confined helium-like) atoms are interesting and simple systems to explore the different aspects of confinement. The complete non-relativistic Hamiltonian in atomic units for the confined helium-like atoms at the center of an impenetrable spherical cavity is given by

$$
\hat{H}=\sum_{i=1}^{2} \hat{h}\left(\mathbf{r}_{i}\right)+V_{e e}\left(\mathbf{r}_{1}, \mathbf{r}_{2}\right),
$$

where $\hat{h}\left(\mathbf{r}_{i}\right)$ is the confined hydrogenic-type Hamiltonian of the $i$-th electron,

$$
\hat{h}\left(\mathbf{r}_{i}\right)=-\frac{1}{2} \nabla_{i}^{2}-\frac{Z}{r_{i}}+V_{c}\left(r_{i}\right)
$$

while $V_{e e}$ corresponds to electrostatic electron-electron repulsion,

$$
V_{e e}\left(\mathbf{r}_{1}, \mathbf{r}_{2}\right)=1 / r_{12}
$$

In above equations, $r_{i}$ represents the distance from the nucleus to the $i$-th electron, $r_{12}$ is the relative distance between the electrons, and the atomic number, $Z=1,2$ and 3, defines the negative hydrogen ion $\left(\mathrm{H}^{-}\right)$, helium (He) and the ionized lithium $\left(\mathrm{Li}^{+}\right)$, respectively. In present work we assume the spherical confining potential as

$$
V_{c}\left(r_{i}\right)=\left\{\begin{array}{c}
0 \text { for } 0<r_{i}<r_{c} \\
\infty \text { for } \quad r_{i} \geq r_{c}
\end{array}\right.
$$

being $r_{c}$ the confinement radius.

Since this is a fermionic system, the electronic total wavefunction has to be antisymmetric by exchange of both electrons due to Pauli exclusion principle. This assumption includes Fermi correlation to the problem. As the Hamiltonian of the problem is spin independent, the product of spatial and spin parts constitute the total wavefunction. Assuming that the spin part is antisymmetrical for the electronic ground state (a singlet electronic state), the spatial part of wavefunction must be symmetrical by exchange of two electrons.

In present work we select three different trial spatial wavefunctions to describe confined helium-like atoms in their ground states in order of better take account of Coulomb correlation. The spin part will be omitted because the Hamiltonian is spin independent.

Our starting point is an exact function for the independent particle model $\varphi_{1}$. The effect of the electrostatic repulsion [Eq. (7)] is regarded as a perturbation in computation of energies. In this independent electron model, the trial wavefunction is given by

$$
\varphi_{1}\left(r_{1}, r_{2}\right)=A e^{\alpha\left(r_{1}+r_{2}\right)} \Omega\left(r_{1}, r_{2}\right),
$$

where the orbital exponent $\alpha$ is adjusted to minimizing the electronic energy from the non-interacting particles Hamiltonian $\hat{H}_{0}=\hat{h}\left(\mathbf{r}_{1}\right)+\hat{h}\left(\mathbf{r}_{2}\right)$. The total ground state energy is found by introducing the electron-electron interaction through the first order perturbation theory. In this case, the one-electron probability density is exactly the same of the confined hydrogenic-type system. In Eq. (9) and the following, $\Omega\left(r_{1}, r_{2}\right)$ is the cutoff function which provides the appropriate boundary conditions.

On the other hand, the complete Hamiltonian [Eq. (5)] is considered in the two other models. The difference between them is how the correlation effect is introduced into the trial wavefunction. In the second model the trial wavefunction $\varphi_{2}$ has a similar analytical expression to the first model, that is,

$$
\varphi_{2}\left(r_{1}, r_{2}\right)=B e^{\beta\left(r_{1}+r_{2}\right)} \Omega\left(r_{1}, r_{2}\right),
$$

but the exponent orbitals $\beta$ is now obtained variationally by minimizing the total electronic energy functional. In this case, an electron interacts with the average field produced by other electron. The apparent effect of each electron on the movement of the other is to reduce the nuclear charge, introducing a change in the probability density when compared to the first model.

The third model considered here consists of introducing an explicit correlation factor between the two electrons in the trial wavefunction, as follows:

$$
\varphi_{3}\left(r_{1}, r_{2}\right)=C e^{\lambda\left(r_{1}+r_{2}\right)} \gamma\left(r_{12}\right) \Omega\left(r_{1}, r_{2}\right),
$$

where the correlation factor $\gamma\left(r_{12}\right)$ is given by

$$
\gamma\left(r_{12}\right)=\left(1+\sigma r_{12}\right) .
$$

The parameters $\lambda$ and $\sigma$ are determined by the variational method. The inclusion of a new variational parameter in $\varphi_{3}$ function makes it more flexible and we expected a wavefunction that better describes the system. In this way, part of correlation energy is recovered by $\varphi_{3}$ function. 
In $\varphi_{1}, \varphi_{2}$ and $\varphi_{3}$ functions, the normalization constants are $A, B$, and $C$, and the cutoff function which provides the appropriate boundary conditions is

$$
\Omega\left(r_{1}, r_{2}\right)=\left[1-\left(\frac{r_{1}}{r_{c}}\right)\right]\left[1-\left(\frac{r_{2}}{r_{c}}\right)\right] .
$$

The Eq. (11) is a correlated wavefunction by $\gamma\left(r_{12}\right)$ factor. The inclusion of this factor introduces considerable complications in problem solution. To solve it, we employ the Hylleraas coordinates [63-66], which are written as

$$
s \equiv r_{2}+r_{1}, t \equiv r_{2}-r_{1} \text { and } u \equiv\left|\mathbf{r}_{1}-\mathbf{r}_{2}\right|
$$

In these coordinates the Hamiltonian of the system is

$$
\begin{aligned}
\widehat{\mathrm{H}}= & -\left(\frac{\partial^{2}}{\partial s^{2}}+\frac{\partial^{2}}{\partial t^{2}}+\frac{\partial^{2}}{\partial u^{2}}\right)-\frac{4 s}{s^{2}-t^{2}} \frac{\partial}{\partial s}+\frac{4 t}{s^{2}-t^{2}} \frac{\partial}{\partial t}-\frac{2}{u} \frac{\partial}{\partial u}- \\
& -2 \frac{s}{u}\left(\frac{u^{2}-t^{2}}{s^{2}-t^{2}}\right) \frac{\partial^{2}}{\partial s \partial u}-2 \frac{t}{u}\left(\frac{u^{2}-s^{2}}{s^{2}-t^{2}}\right) \frac{\partial^{2}}{\partial t \partial u}-4 Z \frac{s}{s^{2}-t^{2}}+\frac{1}{u}
\end{aligned}
$$

The correlated wavefunction is written as

$$
\varphi_{3}(s, t, u)=G e^{\sigma s}(1+b u) \Upsilon(s, t),
$$

the normalization constant is $G$. For the cutoff function we have

$$
\Upsilon(s, t)=\left[r_{c}-\left(\frac{s-t}{2}\right)\right]\left[r_{c}-\left(\frac{s+t}{2}\right)\right]
$$

The volume element in terms of Hylleraas coordinates is

$$
d v=2 \pi^{2}\left(s^{2}-t^{2}\right) u d s d t d u
$$

with the limits of integration being

$$
\begin{aligned}
\int d s d u d t= & \int_{0}^{r_{c}} d s \int_{0}^{s} d u \int_{0}^{u} d t+\int_{r_{c}}^{2 r_{c}} d s \int_{0}^{2 r_{c}-s} d u \int_{0}^{u} d t+ \\
& +\int_{r_{c}}^{2 r_{c}} d s \int_{2 r_{c}-s}^{s} d u \int_{0}^{2 r_{c}-s} d t
\end{aligned}
$$

The Hylleraas coordinates are considered to be coordinate systems of two electrons.

\section{Results and discussions}

In this section, we present and discuss our results for the confined $\mathrm{H}^{-}, \mathrm{He}$ and $\mathrm{Li}^{+}$atoms (ground states energy) under various confinement conditions. In subsect. 3.1 we consider the results of expectation value of the energy. In subsect. 3.2, we determine the informational entropies $S_{r}$ and $S_{p}$ using the Eqs. (2) and (3); and, once get these values, we find the entropy sum $S_{t}$. In subsect. 3.3 we produce an informational study in the strong confinement regime.

We use the softwares Maple13 and QtiPlot to perform the calculations and plot the graphs, respectively. In particular, the numerical calculations, including the numerical integrations, were performed with an accuracy of at least 10 digits. Thus, even with an eventual propagation of numerical errors, we estimate that our results have an accuracy of at least 6 digits, so that the values presented in the tables are accurate within the significant figures presented.

We use the atomic units (a.u.) system. This system uses the electronic mass, $m_{e}$, the elementary charge $e$ of the electron, the constant of electrostatic force, $1 / 4 \pi \varepsilon_{0}$, and the reduced Planck constant, $\hbar$, as standard units of their respective quantities. Here, we consider $m=m_{e}$ for all the calculations. 


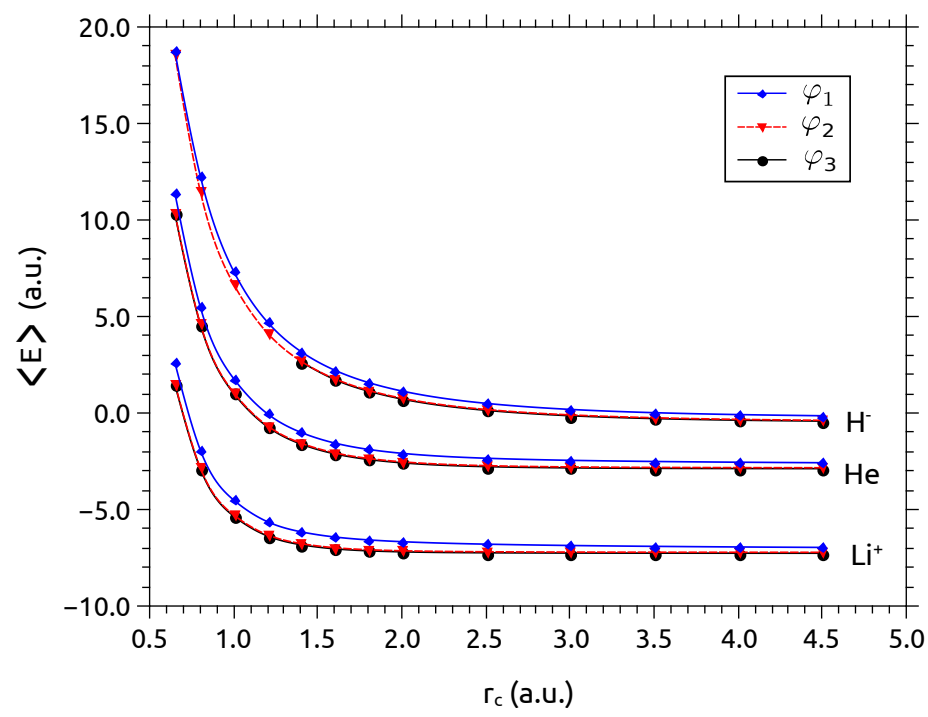

Fig. 1 Values of $\langle E\rangle$ as a function of $r_{c}$ for the confined $\mathrm{H}^{-}, \mathrm{He}$ and $\mathrm{Li}^{+}$atoms in their ground states.

\subsection{Energy analysis}

The expectation value of the energy, $\langle E\rangle$, for the confined $\mathrm{H}^{-}, \mathrm{He}$ and $\mathrm{Li}^{+}$atoms in their ground states for three wavefunctions $\varphi_{1}, \varphi_{2}$ and $\varphi_{3}$, as a function of $r_{c}$, are displayed in Fig. 1 and organized in Table S.1 of the Supporting Information. We determine the $\langle E\rangle$ values of $\varphi_{1}$ adding the values from model independent particles, $\left\langle E^{0}\right\rangle$, plus a first correction, $\left\langle E^{1}\right\rangle$, established by expectation value of the $V_{e e}$ term. Adjusting the variational parameter in $\varphi_{2}$, with the complete Hamiltonian (5), we obtain better energy values than $\varphi_{1}$. Besides, using the $\gamma\left(r_{12}\right)$ term in $\varphi_{3}$, we get the best results in this work, recovering a part of the correlation energy. We detect that the energy values improve in order of consideration of the Coulomb correlation. We can note, from Fig. 1, that $\langle E\rangle$ values tend to the values of the unconfined system (free system) when $r_{c}$ goes to infinity. In another situation, the curves of $\langle E\rangle$ blow up when the value of $r_{c}$ becomes smaller.

The $\langle E\rangle$ values taken by $\varphi_{3}$ for all three isoelectronic members are very close and, occasionally, are lower energies than the results of the Ref. [27] that makes its analysis from density functional theory. For He the energy values provided by $\varphi_{2}$ and $\varphi_{3}$ have a good agreement with the results presented by the Ref. [67], which also performs a variational calculation. Such comparisons indicate that the values of energy obtained here are compatible with the results published in the literature.

The investigation of the correlation effects under different confinement conditions, can be done by difference between the expectation value of the energies $\left\langle E\left[\varphi_{2}\right]\right\rangle$ and $\left\langle E\left[\varphi_{3}\right]\right\rangle$, determined by $\varphi_{2}$ and $\varphi_{3}$, respectively, as follows:

$$
\mu=\left\langle E\left[\varphi_{2}\right]\right\rangle-\left\langle E\left[\varphi_{3}\right]\right\rangle .
$$

The quantity $\mu$ is displayed in Fig. 2 as a function of $r_{c}$. Note that in the atoms studied here the $\mu$ quantity increases with $Z$ increment, and that each $\mu$ associated with a specific confined atomic system has a peak, whose maximum value and position is: the $\mathrm{H}^{-}, \mu^{\max } \approx 0.0429$ at $r_{c} \approx 2.5603$; He, $\mu^{\max } \approx 0.0514$ at $r_{c} \approx 1.8132$; and, finally, for the $\mathrm{Li}^{+}$, $\mu^{\max } \approx 0.0589$ at $r_{c} \approx 1.2765$. For larger $r_{c}$, the $\mu$ values tend to their free atom values. Moreover, when $r_{c}$ goes to zero, the $\mu$ values decrease, indicating that the energy values of $\varphi_{3}$ approaches of $\varphi_{2}$. In the scenario, the correlation factor $\gamma\left(r_{12}\right)$ loses importance on the characterizing of the wavefunction. The $\mu$ values are close to true correlation energy $\left(E_{H F}-E_{\text {exact }}\right)$ for the unconfined or confined two-electrons atoms [67].

One thing to note is about the $\mathrm{H}^{-}$anion. The energy values produced by $\varphi_{1}, \varphi_{2}$ and $\varphi_{3}$ could represent the $\mathrm{H}^{-}$as an unstable arrangement. However, the imposed confinement barriers avoid that one of the electrons detaches from the atom. In the free $\mathrm{H}^{-}$the electrons are loosely attached to the nucleus, so its structure could be energetically appropriate to a dissociation in a neutral hydrogen atom plus an ejected electron. These particularities of the $\mathrm{H}^{-}$can to difficult the study of this ion through the variational method and to produce some numerical instability in the determination of physical quantities of interest in regions of rigorous confinement. Nevertheless, investigations demonstrate that a unique bound state is observed when using more elaborate variational wavefunctions [68-71].

\subsection{Information entropies}

The $S_{r}$ values as a function of $r_{c}$ for the confined $\mathrm{H}^{-}, \mathrm{He}$ and $\mathrm{Li}^{+}$atoms in their ground states are showed in Fig. 3 and organized in Table 1 and Table S2 of the Supporting Information. The first observation is that, for the same $r_{c}$, the informational entropies determined using the trial $\varphi_{1}$ function are exactly twice the informational entropies of equivalent 


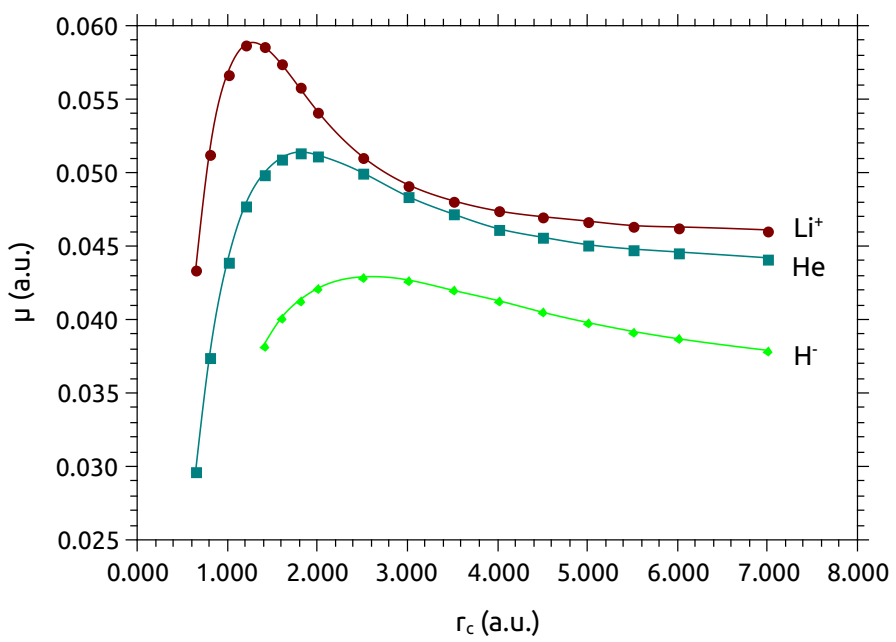

Fig. 2 Values of $\mu$ as a function of $r_{c}$ for the confined $\mathrm{H}^{-}, \mathrm{He}$ and $\mathrm{Li}^{+}$atoms in their ground states.

hydrogenic-like systems obtained previously by us in Ref. [21]. For this comparison, we consider the data calculated using the trial $\phi_{11}^{c}$ function of such publication. The reason for this agreement comes from the fact that its exponential $\alpha$ parameter was obtained disregarding the electron-electron repulsion term. Besides, when $r_{c}$ goes to infinity the $S_{r}$ values tend to the $S_{r}$ values of the unconfined systems presented in Ref. [26], indicating the validity of our results. It should be noted that, in their work, Nasser et al. used wavefunctions with the same functional forms of $\varphi_{2}$ and $\varphi_{3}$, excluding the cutoff function, to treat the free two-electron helium-like atoms.

Table 1 Values of $S_{r}$ as a function of $r_{c}$ for the $\mathrm{H}^{-}, \mathrm{He}$ and $\mathrm{Li}^{+}$atoms in their ground states using the functions $\varphi_{1}, \varphi_{2}$ and $\varphi_{3}$. Values of $r_{c}$ in atomic units system. The literature references are given as superscript.

\begin{tabular}{|c|c|c|c|c|c|c|c|c|c|}
\hline & \multicolumn{3}{|c|}{$\mathrm{H}^{-}$} & \multicolumn{3}{|c|}{$\mathrm{He}$} & \multicolumn{3}{|c|}{$\mathrm{Li}^{+}$} \\
\hline$r_{c}$ & $\varphi_{1}$ & $\varphi_{2}$ & $\varphi_{3}$ & $\varphi_{1}$ & $\varphi_{2}$ & $\varphi_{3}$ & $\varphi_{1}$ & $\varphi_{2}$ & $\varphi_{3}$ \\
\hline 1.0000 & 1.0823 & 1.1760 & 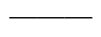 & 0.6345 & 0.7734 & 0.7779 & -0.0029 & 0.1973 & 0.2071 \\
\hline 2.0000 & 4.7933 & 5.0561 & 5.0543 & 3.3254 & 3.8232 & 3.8400 & 1.4804 & 2.0257 & 2.0578 \\
\hline 4.0000 & 7.4843 & 8.4092 & 8.3985 & 4.0407 & 4.9813 & 5.0173 & 1.6670 & 2.3137 & 2.3514 \\
\hline 6.0000 & 8.0720 & 9.7039 & 9.6810 & 4.0998 & 5.0960 & 5.1336 & 1.6860 & 2.3413 & 2.3793 \\
\hline 10.0000 & 8.2406 & 10.3519 & 10.3056 & 4.1212 & 5.1345 & 5.1724 & 1.6939 & 2.3525 & 2.3906 \\
\hline free & - & $10.538^{\mathrm{a}}$ & $10.479^{\mathrm{a}}$ & - & $5.1500^{\mathrm{a}}$ & $5.1881^{\mathrm{a}}$ & 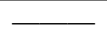 & $2.3577^{\mathrm{a}}$ & $2.3959^{a}$ \\
\hline
\end{tabular}

From graph (a) of the Fig. 3, we see that the difference between $S_{r}$ values determined by $\varphi_{1}$ and $\varphi_{2}$ are higher than $\varphi_{2}$ and $\varphi_{3}$. The $S_{r}$ curves obtained by $\varphi_{2}$ and $\varphi_{3}$ are close to producing an overlap in such figure. Moreover, when the confinement increases the $S_{r}$ curves tend to the negative values. In Table 1 and Table $\mathrm{S} 2$ of the Supporting Information, the results of $S_{r}$ for the $\mathrm{He}$ and $\mathrm{Li}^{+}$atoms increase using the functions $\varphi_{1}, \varphi_{2}$ and $\varphi_{3}$, in this order. For the $\mathrm{H}^{-}$, the $S_{r}$ values determined by $\varphi_{1}$ are less than those employing $\varphi_{2}$, whereas the calculated by $\varphi_{2}$ are greater than those provided by $\varphi_{3}$. This phenomenon occurs, in $\mathrm{H}^{-}$, because the use of $\gamma\left(r_{12}\right)$ induces the decoupling of an electron from atom, while the other get closer of the nucleus. This effect produces a more localized probability density in position space and, consequently, a lower value for $S_{r}$ using $\varphi_{3}$.

Also based on the graph (a) of the Fig. 3 note that the values of $S_{r}$ can be negative. These values correspond to regions where the probability densities are highly localized. This fact can also be observed in one-dimensional systems [14], in the spherically confined hydrogen atom [21] and systems with static screened Coulomb potential [72]. This result has a simple explanation in the quantum context [73]: when $r_{c}$ is reasonably small, the probability density becomes large and $a_{0}^{6} \rho\left(\mathbf{r}_{1}, \mathbf{r}_{2}\right)>1$. In this situation, $-\rho\left(\mathbf{r}_{1}, \mathbf{r}_{2}\right) \ln \left(a_{0}^{6} \rho\left(\mathbf{r}_{1}, \mathbf{r}_{2}\right)\right)<0$ and, then, $S_{r}$ may be negative.

We visualize the performance of the $S_{p}$ versus $r_{c}$ curves for $\varphi_{1}$ and $\varphi_{2}$ in the graph (b) of the Fig. 3 and we organize the numerical values in Table 2 and Table S3 of the Supporting Information. The incorporation of the Coulomb correlation on the wavefunction $\varphi_{2}$ causes a reduction in the $S_{p}$ values. When $r_{c}$ goes to infinity the $S_{p}$ values pass to constant values. In another situation, when the confinement increases, an upward inflection of the curves is identified. The integral calculations of the information entropy on momentum space showed numerical instability and were truncated to a given value of the integration limit. Taking that into account, we guarantee the accuracy of the results obtained of $S_{p}$ using the 
(a)

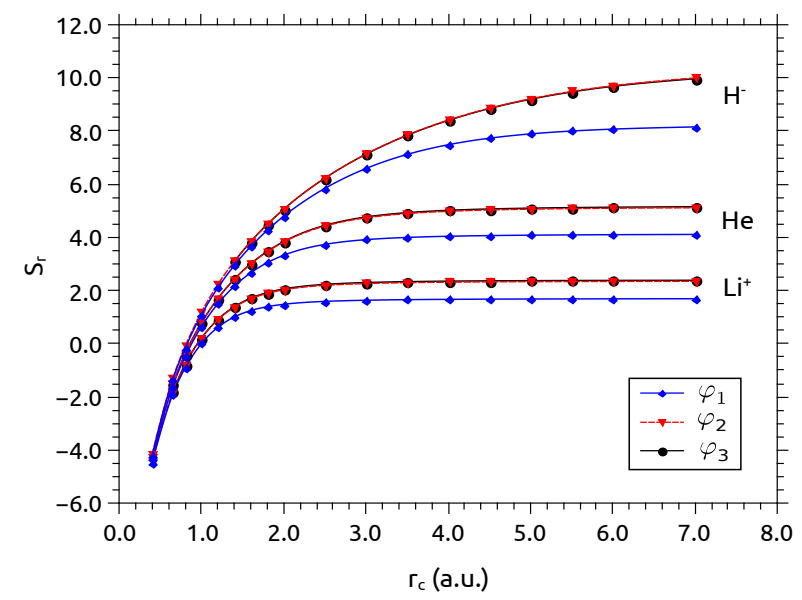

(b)

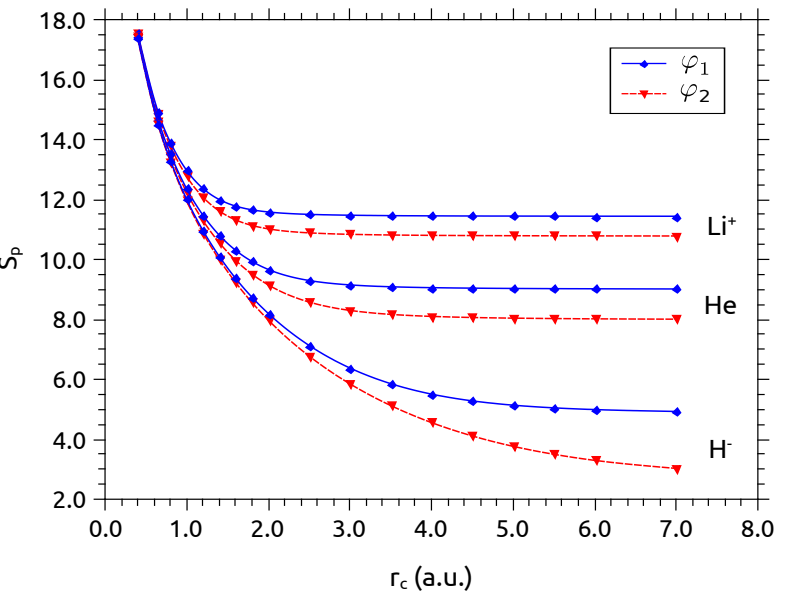

Fig. 3 Values of $S_{r}$ and $S_{p}$ as a function of $r_{c}$ for the confined $\mathrm{H}^{-}, \mathrm{He}$ and $\mathrm{Li}^{+}$atoms in their ground states.

functions $\varphi_{1}$ and $\varphi_{2}$. Studies involving Fourier transforms in correlated wavefunctions have been started [74], allowing us in the future to provide the $S_{p}$ values for the function $\varphi_{3}$.

Table 2 Values of $S_{p}$ as a function of $r_{c}$ for the $\mathrm{H}^{-}, \mathrm{He}$ and $\mathrm{Li}^{+}$atoms in their ground states using the functions $\varphi_{1}$ and $\varphi_{2}$. Values of $r_{c}$ in atomic units system.

\begin{tabular}{c|cc|cc|cc}
\hline & \multicolumn{2}{|c|}{$\mathrm{H}^{-}$} & \multicolumn{2}{c|}{$\mathrm{He}$} & \multicolumn{2}{c}{$\mathrm{Li}^{+}$} \\
\hline$r_{c}$ & $\varphi_{1}$ & $\varphi_{2}$ & $\varphi_{1}$ & $\varphi_{2}$ & $\varphi_{1}$ & $\varphi_{2}$ \\
\hline 1.0000 & 12.0076 & 11.9496 & 12.3575 & 12.2374 & 12.9705 & 12.7709 \\
\hline 2.0000 & 8.1986 & 7.9796 & 9.6665 & 9.1507 & 11.5832 & 11.0197 \\
\hline 4.0000 & 5.5076 & 4.5581 & 9.0582 & 8.0998 & 11.4537 & 10.8032 \\
\hline 6.0000 & 4.9915 & 3.2971 & 9.0209 & 8.0183 & 11.4424 & 10.7857 \\
\hline 10.0000 & 4.8731 & 2.7320 & 9.0081 & 7.9931 & 11.4377 & 10.7787 \\
\hline
\end{tabular}

The Ref. [75] helps us to understand the behavior of $S_{r}$ and $S_{p}$ with the $Z$ variation. The increase of the atomic number produces lower results of the expectation values of $r_{1}$ and $r_{12}$, making more localized systems, consequently the $S_{r}$ values are reduced with $Z$ increment in Table 1 and Table S2 of the Supporting Information. On the other hand, the reduction of the atomic number makes weaker interactions. The movement of particles is less chaotic, then the $S_{p}$ values decrease with $Z$ reduction in Table 2 and Table S3 of the Supporting Information.

The $S_{t}$ values as a function of $r_{c}$ for the confined helium-like atoms in their ground states are organized in Table 3 and Table $\mathrm{S} 4$ of the Supporting Information and we display in Fig. 4 the overall behavior of it. Note that the entropic uncertainty relation $\left(S_{t} \geq 12.8684\right)$ is satisfied for all studied confined systems. Moreover, both results obtained with $\varphi_{1}$ and $\varphi_{2}$ trial wavefunctions for the three helium-like atoms show the same overall behavior. All $S_{t}$ curves have a minimum for intermediate values of $r_{c}$. In addition, for large $r_{c}$, the values of $S_{t}$ using $\varphi_{1}$ and $\varphi_{2}$, for the confined $\mathrm{H}^{-}, \mathrm{He}$ and $\mathrm{Li}^{+}$ atoms tend towards the value of 13.1332 of the free atomic system. And in the direction of small $r_{c}$, the entropy sums present an increase in their values. As we have observed in subsect. 2.1 the entropy sum gives rise to entropic uncertainty relation. In this context, at the point where $S_{t}$ has its lowest value $\left(S_{t}^{\min }\right)$ the system is on the situation of lower entropic uncertainty.

One interesting aspect to point out is that the values of the confinement radius, $r_{c}^{\text {min }}$, in which $S_{t}^{\min }$ arise, are shifted to larger values in order of better take account of Coulomb correlation in wavefunction. For the $\mathrm{H}^{-}$using $\varphi_{1}$ we have $S_{t}^{\min }=12.9676$ in $r_{c}^{\min }=3.0010$ u.a., while for $\varphi_{2}$ we have $S_{t}^{\min }=12.9673$ in $r_{c}^{\min }=4.0000$ u.a.. For the He, we have for $\varphi_{1}$ the value of $S_{t}^{\min }=12.9675$ in $r_{c}^{\min }=1.4001$ and for $\varphi_{2}$ we have $S_{t}^{\min }=12.9679$ in $r_{c}^{\min }=1.6000$ u.a.. For the $\mathrm{Li}^{+}$, we have for $\varphi_{1}$ the value of $S_{t}^{\min }=12.9674$ arise in $r_{c}^{\min }=0.9500$ u.a. and for $\varphi_{2}$ we have $S_{t}^{\min }=12.9675$ in $r_{c}^{\min }=1.1000$ a.u.. So, the inclusion of the Coulomb correlation modifies, in some way, the balance between Coulomb and confining potential, modifying the confinement radius associated with the lowest entropy sum. Differences in $r_{c}^{\mathrm{min}}$ due to improved model have a greater impact on $\mathrm{H}^{-}$anion, reinforcing its intriguing characteristics.

In the context of the density functional theory $[76,77]$ the informational study of confined two-electron systems has been done utilizing the one-electron density normalized to unity [27, 28]. Nevertheless, analysis using two-electron probability densities normalized to unity, as in the present work, are also presented in the literature involving free helium- 
Table 3 Values of $S_{t}$ as a function of $r_{c}$ for the $\mathrm{H}^{-}, \mathrm{He}$ and $\mathrm{Li}^{+}$atoms in their ground states using the functions $\varphi_{1}$ and $\varphi_{2}$. Values of $r_{c}$ in atomic units system.

\begin{tabular}{c|cc|cc|cc}
\hline & \multicolumn{2}{|c|}{$\mathrm{H}^{-}$} & \multicolumn{2}{c|}{$\mathrm{He}$} & \multicolumn{2}{c}{$\mathrm{Li}^{+}$} \\
\hline$r_{c}$ & $\varphi_{1}$ & $\varphi_{2}$ & $\varphi_{1}$ & $\varphi_{2}$ & $\varphi_{1}$ & $\varphi_{2}$ \\
\hline 1.0000 & 13.0899 & 13.1256 & 12.9919 & 13.0107 & 12.9676 & 12.9682 \\
\hline 2.0000 & 12.9919 & 13.0357 & 12.9919 & 12.9739 & 13.0636 & 13.0454 \\
\hline 4.0000 & 12.9919 & 12.9673 & 13.0989 & 13.0811 & 13.1207 & 13.1169 \\
\hline 6.0000 & 13.0636 & 13.0010 & 13.1207 & 13.1143 & 13.1284 & 13.1270 \\
\hline 10.0000 & 13.1137 & 13.0839 & 13.1294 & 13.1276 & 13.1316 & 13.1312 \\
\hline
\end{tabular}

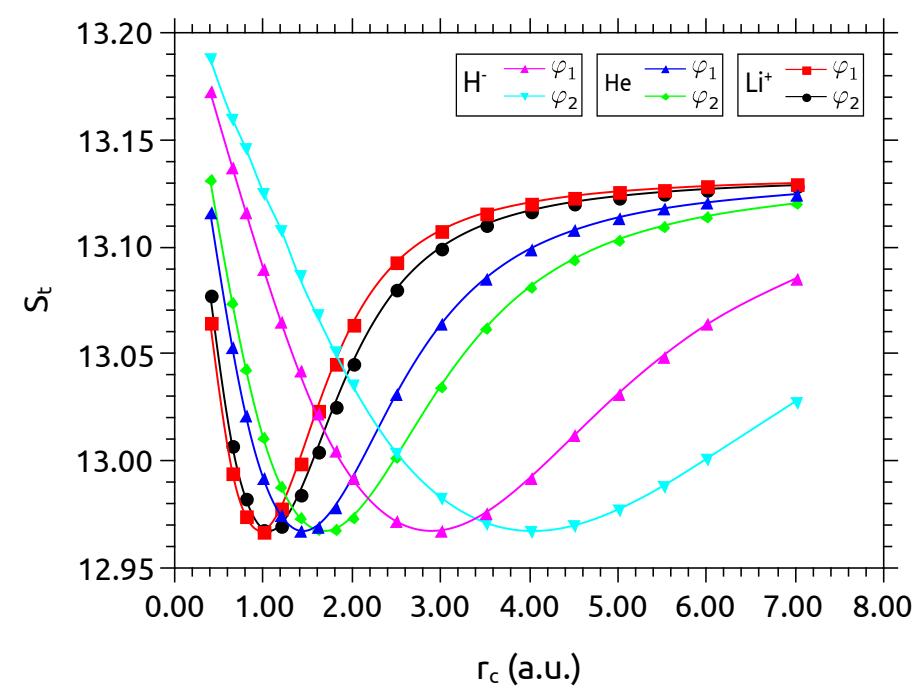

Fig. 4 Values of $S_{t}$ as a function of $r_{c}$ for the confined $\mathrm{H}^{-}, \mathrm{He}$ and $\mathrm{Li}^{+}$atoms in their ground states .

like atoms [25, 26]. For more details on the different entropic formulations and how they can be contrasted see Ref. [78]. Lastly, we did not find $S_{r}, S_{p}$ and $S_{t}$ available entropies values in literature for confined helium-like atoms using twoelectron probability densities normalized to unity and written in terms of two-electron wavefunctions to compare with our results.

\subsection{Strong confinement regime}

The strong or extreme confinement regime for confined atomic system can be defined when the influence of the confining potential becomes greater than the free atomic system potential to specific configurations (or $r_{c}$ values). One important characteristic of the strong confinement region is that the probability density is highly concentrated in position space, distinctly to weakly confined regions. The definition of specific radii for the strong confinement regime in two-electrons atoms can be based on the ionization radius [79-81]. In our previous studies of confined harmonic oscillator [51] and confined hydrogenic-like atoms [21], we have suggested that the entropy sum can be employed to determine a strong or rigorous confinement regime.

In the present work, based on the entropy sum we define three regions of confinement for the systems. In the strong confinement region, when $r_{c} \rightarrow 0$, the confinement potential is preponderant. On the other hand, the weak confinement correspond to the regions where $r_{c} \rightarrow \infty$ and the Coulomb potential is dominant. The intermediate confinement region is close of the value of $S_{t}^{\min }$ where the effects of the confining and Coulomb potentials has approximately a similar impact or influence on the system (the confinement and Coulomb potentials are compensated).

To illustrate and define more precisely the confinement regimes we use the values of $S_{t}$, obtained by $\varphi_{2}$ function, to plot the graph of the entropy sum for the $\mathrm{H}^{-}, \mathrm{He}$ and $\mathrm{Li}^{+}$atoms in Fig. 5. The intermediate confinement region for each atom studied is defined between the values of $r_{c}$ where the horizontal line crosses the curve of $S_{t}$ versus $r_{c}$. The horizontal line marks the half well depth of the entropy curve, being given by

$$
\sigma_{[a t o m s]}=\frac{S_{t}\left[r_{c}=\infty\right]-S_{t}\left[r_{c}^{\min }\right]}{2}
$$

where the $S_{t}$ values are calculated in $r_{c}$ tending to infinity and in $r_{c}^{\min }$ ( $r_{c}$ value where $S_{t}^{\min }$ occurs). The use of $\sigma_{[a t o m s]}$ provides a good approximation for the intermediate confinement. As defined by Eq. (21), the main graph of Fig. 5 
have horizontal lines referring to $\mathrm{H}^{-}, \mathrm{He}$ and $\mathrm{Li}^{+}$atoms, however due to the graphic scale these lines overlap. In the secondary graphic of the same figure we highlight a portion in zoom of the main graph contrasting the emergence of the three horizontal lines.

In addition, in Fig. 5 the strong and weak confinement correspond to the regions, respectively, left $\left(r_{c} \rightarrow 0\right)$ and right $\left(r_{c} \rightarrow \infty\right)$, of the intermediate confinement. Based on this analysis in Table 4 we organize the numerical values that limit the confinement regions and height of half well depth of the atoms studied.

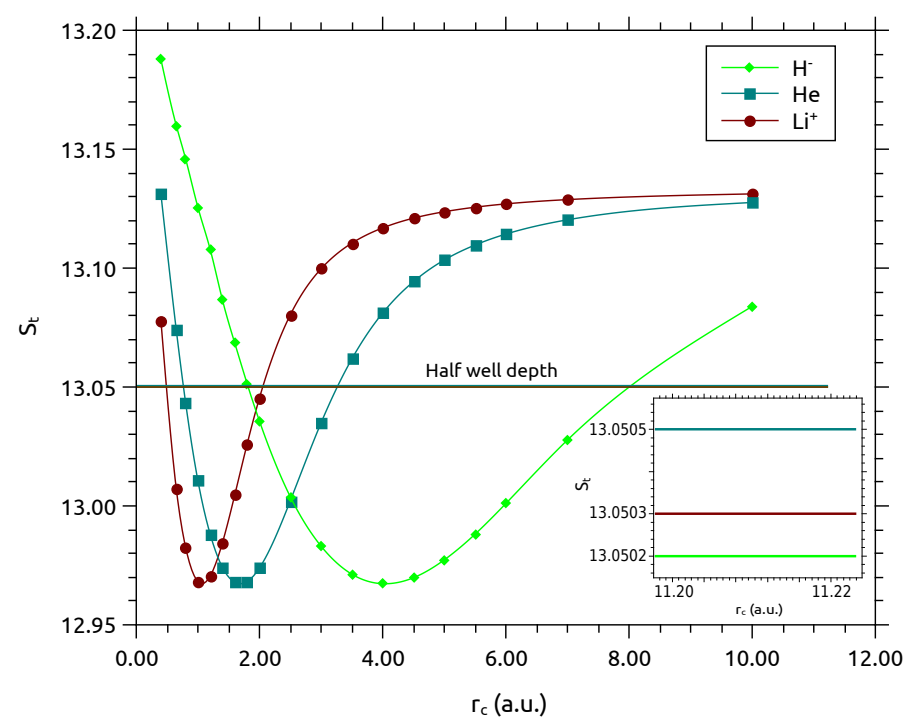

Fig. 5 Values of $S_{t}$ as a function of $r_{c}$ for the confined $\mathrm{H}^{-}, \mathrm{He}$ and $\mathrm{Li}^{+}$atoms in their ground states. $S_{t}$ values using the $\varphi_{2}$ function. In the secondary graphic we highlight a portion in zoom of the main graph contrasting the emergence of the three horizontal lines.

Table 4 Confinement region of the confined two-electrons atoms. Values of $r_{c}$ in atomic units system.

\begin{tabular}{|c|c|c|c|c|}
\hline Atoms & height of half well depth & Strong & Intermediate & Weak \\
\hline $\mathrm{H}^{-}$ & 13.0502 & $r_{c}<1.80$ & $1.80 \leq r_{c} \leq 8.00$ & $r_{c}>8.00$ \\
\hline $\mathrm{He}$ & 13.0505 & $r_{c}<0.75$ & $0.75 \leq r_{c} \leq 3.25$ & $r_{c}>3.25$ \\
\hline $\mathrm{Li}^{+}$ & 13.0503 & $r_{c}<0.50$ & $0.50 \leq r_{c} \leq 2.10$ & $r_{c}>2.10$ \\
\hline
\end{tabular}

Some of the main aspects of the strong or extreme confinement regime is the fact that, in this region, the effects of electronic correlation become essentially null, however the influence of the confining potential becomes greater than Coulomb potential. In this specific region, the system have the probability density highly concentrated in position space $[82,83]$. These characteristics are obtained in the present work through an elegant informational analysis.

Initially, we examine the inclusion of the $V_{e e}$ term in Hamiltonian (5) through the quantity $\Lambda$, represented in terms of the entropy sums been determined by $\varphi_{1}$ and $\varphi_{2}$ functions, as follows:

$$
\Lambda=\frac{S_{t}\left[\varphi_{1}\right]}{S_{t}\left[\varphi_{2}\right]}
$$

In addition, using the $S_{r}$ and $S_{p}$ entropies, computed also by $\varphi_{1}$ and $\varphi_{2}$, we have established the $\beta_{r}$ and $\beta_{p}$ quantities, that is,

$$
\beta_{r}=S_{r}\left[\varphi_{1}\right]-S_{r}\left[\varphi_{2}\right]
$$

and

$$
\beta_{p}=S_{p}\left[\varphi_{1}\right]-S_{p}\left[\varphi_{2}\right] .
$$

In Figs. 6, we present the curves of $\Lambda, \beta_{r}$ and $\beta_{p}$ as a function of $r_{c}$ for the examined atoms, respectively in graphs (a), (b) and (c). In situation (a) the $\Lambda$ values tend to unity when the $r_{c}$ values decrease. The graphs (b) and (c) indicate that the $\beta_{r}$ and $\beta_{p}$ values goes to zero when the confinement situation intensifies. These results imply that when $r_{c}$ goes to zero, the entropy values related to $\varphi_{2}$ function, in position space or in momentum space, tend to entropy values related to $\varphi_{1}$ function. Therefore, we conclude that the independent particle model can be successfully employed in the strong confinement region. 
(a)

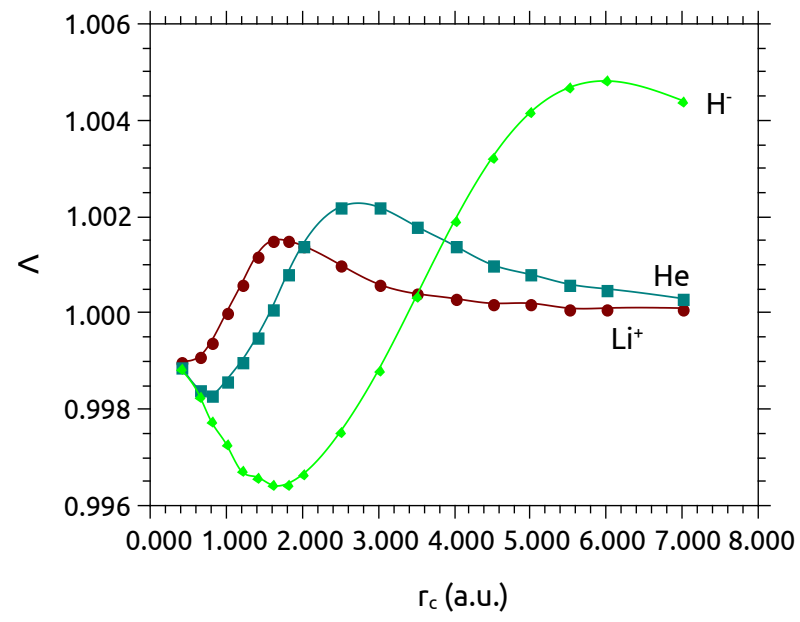

(b)

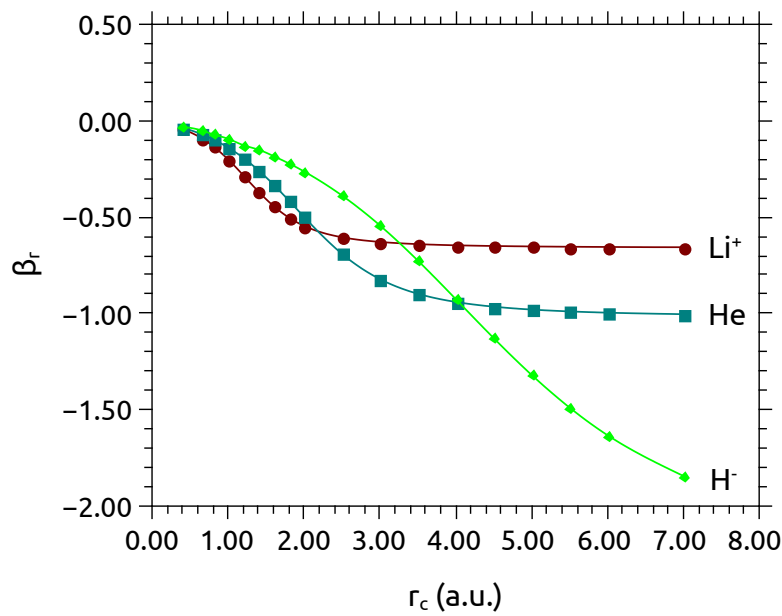

(c)

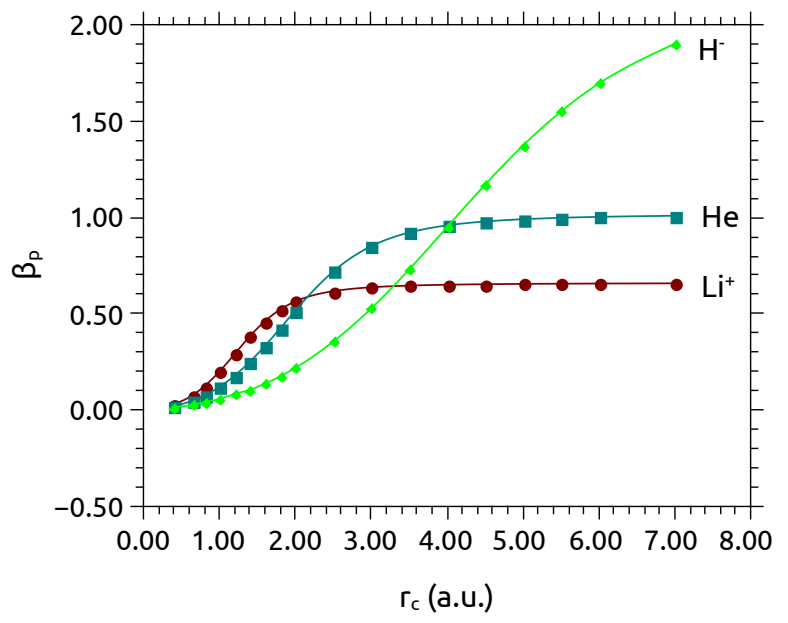

Fig. 6 Values of $\Lambda, \beta_{r}$ and $\beta_{p}$ as a function of $r_{c}$ for the confined $\mathrm{H}^{-}, \mathrm{He}$ and $\mathrm{Li}^{+}$atoms in their ground states.

The values of the entropy sum of the investigated confined two-electrons atoms have interesting relation with our previous researches involving the confined hydrogenic-like atoms $\left(\mathrm{He}^{+}, \mathrm{H}\right.$ and $\left.\mathrm{Li}^{++}\right)$accessible in Ref. [21]. For instance, for $r_{c}=0.4000$ a.u., we have the values of $S_{t}\left[\mathrm{H}^{-}\right]=13.1878, S_{t}[\mathrm{He}]=13.1312$ and $S_{t}\left[\mathrm{Li}^{+}\right]=13.0780$. Moreover, $2 S_{t}[\mathrm{H}]=13.1666,2 S_{t}\left[\mathrm{He}^{+}\right]=13.1072$ and $2 S_{t}\left[\mathrm{Li}^{++}\right]=13.0518$. The numerical results show that the $S_{t}$ values of $\mathrm{H}^{-}$, $\mathrm{He}$ and $\mathrm{Li}^{+}$are approximately the double of $\mathrm{H}, \mathrm{He}^{+}$, and $\mathrm{Li}^{++}$, respectively. This investigation denotes the loss of importance of the $V_{e e}$ term for small confinement radii, being in favor of the adoption of the independent particle model.

We utilize the $2 e^{-}$system to auxiliary our investigation of the strong confinement region. This system is constituted by two non-interacting electrons confined by a impenetrable spherical cage (see Appendix). In this sense, we determine the quantity $\Gamma$ as follows:

$$
\Gamma_{[a t o m s]}=S_{r}\left[2 e^{-}\right]-S_{r}[\text { atoms }] .
$$

The $S_{r}$ [atoms] values are obtained employing the $\varphi_{2}$ function, where the reported atoms are $\mathrm{H}^{-}, \mathrm{He}$ and $\mathrm{Li}^{+}$. In Fig. 7 , $\Gamma_{[a t o m s]}$ versus $r_{c}$ curves, we note that increasing the confinement effect the $\Gamma_{[a t o m s]}$ values go to zero. That is, the $S_{r}$ values of $\mathrm{H}^{-}, \mathrm{He}$ and $\mathrm{Li}^{+}$tend for the results of $2 e^{-}$, which leads us to establish that when $r_{c}$ go to zero the confining potential becomes more relevant than the nuclear potential. Furthermore, the the effects of Coulomb correlation become negligible. These effects favor employment of the model of non-interacting electrons inside a sphere for the confined two-electrons atoms in the strong confinement regime.

The $S_{r}$ values obtained for the systems $2 e^{-}, \mathrm{H}^{-}, \mathrm{He}$ and $\mathrm{Li}^{+}$have the following relation: $S_{r}\left[2 e^{-}\right]>S_{r}\left[\mathrm{H}^{-}\right]>S_{r}[\mathrm{He}]>$ $S_{r}\left[\mathrm{Li}^{+}\right]$. Besides, in $r_{c}=0.4000$ a.u., we have the values approximately equal of $S_{t}\left[2 e^{-}\right]=13.2344, S_{t}\left[\mathrm{H}^{-}\right]=13.1878$, $S_{t}[\mathrm{He}]=13.1312$ and $S_{t}\left[\mathrm{Li}^{+}\right]=13.0780$. Thus, we infer that in strong confinement the electrons are primarily bound to the confinement potential, instead of the nuclear potential, and the effects of electron-electron interaction lose importance. We found similar conclusion analyzing the Fig. 7. For the $2 e^{-}$system the values of the entropic quantities and of the expectation value of the energy are available in Table S5 of the Supporting Information. 


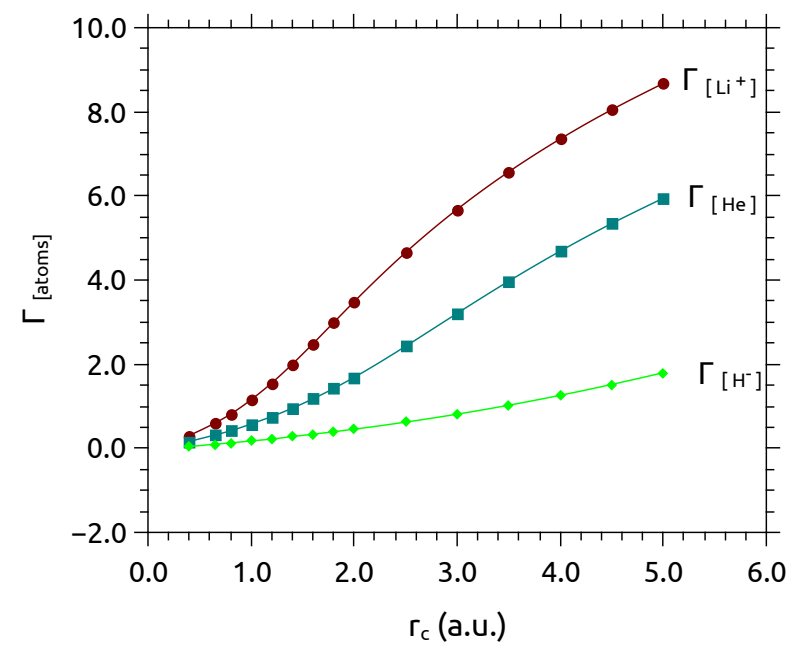

Fig. 7 Values of $\Gamma_{[a t o m s]}$ as a function of $r_{c}$ for the confined $\mathrm{H}^{-}, \mathrm{He}$ and $\mathrm{Li}^{+}$atoms in their ground states.

\section{Conclusion}

In this work, we determined the expectation values of the energy, $\langle E\rangle$, and the informational quantities $S_{r}, S_{p}$ and $S_{t}$, for the confined $\mathrm{H}^{-}, \mathrm{He}$ and $\mathrm{Li}^{+}$atoms (ground states) confined at the centre of an impenetrable spherical cavity. The informational quantities were calculated using the two-electron probability densities normalized to unity. For this, we have employed three different variational wavefunctions.

We obtained more accurate results of $\langle E\rangle$ using the wavefunctions that better take account of Coulomb correlation. The correlation term $\gamma\left(r_{12}\right)$ goes losing importance when the confinement increases. The $\langle E\rangle$ values tend to the results of the unconfined system when $r_{c}$ goes to infinity. On the other hand, the energy values experience a considerable increase when $r_{c}$ value diminishes. The obtained $\langle E\rangle$ values have a good agreement with found results in literature.

The $S_{r}$ values increase, for the confined $\mathrm{He}$ and $\mathrm{Li}^{+}$atoms, using the $\varphi_{1}, \varphi_{2}$ and $\varphi_{3}$ wavefunctions, respectively. For $\mathrm{H}^{-}$, the $S_{r}$ values increase of $\varphi_{1}$ to $\varphi_{2}$ and decreases of $\varphi_{2}$ to $\varphi_{3}$. This last effect occurs because in $\varphi_{3}$ we have a more localized probability density. The $S_{p}$ values decrease and the values of $r_{c}$, in which $S_{t}^{\text {min }}$ arise, is shifted in order of better take account of Coulomb correlation in the system. When $r_{c}$ value goes to infinity the $S_{r}, S_{p}$ and $S_{t}$ values go to the results of the unconfined system. The $S_{p}$ values using the function $\varphi_{3}$ have not been determined, fortunately this not produces changes on the main conclusions of our study. In the future we will return to this specific question.

All the minimum values of $S_{t}$ obtained here satisfy the entropy uncertainty relation. We identified a situation of lower entropic uncertainty in $S_{t}^{\min }$ where the Coulomb and the confining potentials are compensated. The symmetry $r_{c}^{m i m} \equiv 3.0 / Z$ a.u found for the two-electrons atoms using the independent particle model, $\varphi_{1}$, is broken with the incorporation of a part of the Coulomb correlation in $\varphi_{2}$ function.

Finally, based on the $S_{t}$ values, we define the regions of strong $\left(r_{c} \rightarrow 0\right)$, intermediate (close of $\left.S_{t}^{\min }\right)$ and weak confinement $\left(r_{c} \rightarrow \infty\right)$ for the confined $\mathrm{H}^{-}, \mathrm{He}$ and $\mathrm{Li}^{+}$atoms in their ground energy states. Analyzing the informational quantities, we concluded that in the strong confinement regime, the effects of Coulomb correlation become negligible. So, we can successfully employ the models of independent particle and two non-interacting electrons confined by a impenetrable spherical cage for the atoms of two electrons when the confinement effects are extreme.

Acknowledgements This work has been supported by the Brazilian agencies CAPES (Coordenação de Aperfeiçoamento de Pessoal de Nível Superior) and CNPq (Conselho Nacional de Desenvolvimento Científico e Tecnológico) through grants to the authors. The authors thank the referees for careful reading of the manuscript and for helpful comments and suggestions.

Author contribution: All authors contributed equally to the paper.

Supporting Information. This manuscript contains supplementary information. Click here to access.

\section{Appendix A:}

The $2 e^{-}$system consists of two non-interacting electrons confined in an impenetrable spherical cage. The non-relativistic Hamiltonian in atomic units for one electron confined in an impenetrable spherical cage, $e^{-}$, is

$$
\hat{H}=-\frac{1}{2} \nabla^{2}+V_{c}(r)
$$


The confining potential is defined as

$$
V_{c}(r)=\left\{\begin{array}{l}
0 \text { for } 0<r<r_{c} \\
\infty \text { for } \quad r \geq r_{c}
\end{array},\right.
$$

being $r_{c}$ the confinement radius. The ground state wavefunction is

$$
\phi(r)=A\left[\frac{\sin \left(\pi r / r_{c}\right)}{r}\right]\left(\frac{1}{4 \pi}\right)
$$

where $A$ is a normalization constant. In this background, for the $2 e^{-}$system the expectation values of the energy, beyond the entropic quantities, are the double of the values obtained for the $e^{-}$system. The $S_{r}, S_{p}, S_{t}$ and $\langle E\rangle$ values as a function of $r_{c}$ for the $2 e^{-}$system in ground state are available in Table S5 of the Supplementary Material.

\section{References}

1. P. Fulde, Electron Correlation in Molecules and Solids (Springer, Berlin, 1995). DOI 10.1007/978-3-642-57809-0

2. R.P. Sagar, H.G. Laguna, N.L. Guevara, Chem. Phys. Lett. 514, 352 (2011). DOI 10.1016/j.cplett.2011.08.032

3. D.P. Tew, W. Klopper, T. Helgaker, J. Comp. Chem. 28, 1307 (2007). DOI 10.1002/jcc.20581

4. L. Rincon, F.J. Torres, M. Becerra, S. Liu, A. Fritsch, R. Almeida, Mol. Phys. 117(5), 610 (2019). DOI 10.1080/ 00268976.2018 .1530462

5. P.O. Löwdin, Adv. Chem. Phys. 2, 207 (1958). DOI 10.1002/9780470143483.ch8

6. A.L. Baskerville, A.W. King, H. Cox, R. Soc. opens ci. 6, 181357 (2019). DOI 10.1098/rsos.181357

7. A. Mohajeri, M. Alipour, J. Mol. Struct.: THEOCHEM 907, 115 (2009). DOI 10.1016/j.theochem.2009.04.036

8. S.P. McCarthy, A.J. Thakkar, Chem. Phys. Lett. 494, 312 (2010). DOI 10.1016/j.cplett.2010.05.095

9. O. Rioul, Théorie de l'information et du codage (Lavoisier, Paris, 2007)

10. C. Shannon, Bell Syst. Tech. J. 27, 379 (1948). DOI 10.1002/j.1538-7305.1948.tb01338.x

11. Z.X. Wang, L.Y. He, D.D. Li, Energy Policy 132, 429 (2019). DOI 10.1016/j.enpol.2019.06.002

12. Z. Liu, P. Shang, Phys. A (Amsterdam, Neth.) 505, 1170 (2018). DOI 10.1016/j.physa.2018.04.041

13. O. Olendski, Ann. Phys. (Berlin) 530, 1700324 (2018). DOI 10.1002/andp.201700324

14. W.S. Nascimento, M.M. de Almeida, F.V. Prudente, Eur. J. Phys. 41(2), 025405 (2020). DOI 10.1088/1361-6404/ ab5f7d

15. A. Ghosal, N. Mukherjee, A.K. Roy, Ann. Phys. (Berlin) 528(11-12), 796 (2016). DOI 10.1002/andp.201600121

16. H.G. Laguna, R.P. Sagar, Ann. Phys. (Berlin) 526(11-12), 555 (2014). DOI 10.1002/andp. 201400156

17. G.H. Sun, S.H. Dong, N. Saad, Ann. Phys. (Berlin) 525(12), 934 (2013). DOI 10.1002/andp.201300089

18. C.R. Estañón, N. Aquino, D. D. Puertas-Centeno, J.S. Dehesa, Int. J. Quantum Chem. 120(11), e26192 (2020). DOI 10.1002/qua.26192

19. N. Mukherjee, A.K. Roy, Eur. Phys. J. D 72, 118 (2018). DOI 10.1140/epjd/e2018-90104-1

20. M.A. Martínez-Sánchez, R. Vargas, J. Garza, Quantum Rep. 1, 208 (2019). DOI 10.3390/quantum1020018

21. W.S. Nascimento, F.V. Prudente, Chem. Phys. Lett. 691, 401 (2018). DOI 10.1016/j.cplett.2017.11.048

22. S.J.C. Salazar, H.G. Laguna, V. Prasad, R.P. Sagar, Int. J. Quantum Chem. 120(11), e26188 (2020). DOI 10.1002/ qua. 26188

23. J.S. Dehesa, E.D. Belega, I.V. Toranzo, A.I. Aptekarev, Int. J. Quantum Chem. 119(18), e25977 (2019). DOI https://doi.org/10.1002/qua.25977

24. J.H. Ou, Y.K. Ho, Int. J. Quantum Chem. 119(14), e25928 (2019). DOI 10.1002/qua.25928

25. I. Nasser, M. Zeama, A. A.-Hady, Mol. Phys. 118(3), 1612105 (2020). DOI 10.1080/00268976.2019.1612105

26. I. Nasser, M. Zeama, A. A.-Hady, Results Phys. 7, 3892 (2017). DOI 10.1016/j.rinp.2017.10.013

27. K.D. Sen, J. Chem. Phys. 123, 074110 (2005). DOI 10.1063/1.2008212

28. S. Majumdar, A. Roy, Quantum Rep. 2, 189 (2020). DOI 10.3390/quantum2010012

29. C.H. Lin, Y.K. Ho, Chem. Phys. Lett. 689, 116 (2017). DOI 10.1016/j.cplett.2017.10.007

30. J.H.O. Lin, Y.K. Ho, Atoms 5, 15 (2017). DOI 10.3390/atoms5020015

31. I. Nasser, M. Zeama, A. Abdel-Hady, Phys. Scr. 95(9), 095401 (2020). DOI 10.1088/1402-4896/abaa09

32. C. Martínez-Flores, Phys. Lett. A 386, 126988 (2021). DOI 10.1016/j.physleta.2020.126988

33. C. Martínez-Flores, M. Martínez-Sánchez, R. Vargas, J. Garza, Eur. Phys. J. D 75, 100 (2021). DOI 10.1140/epjd/ s10053-021-00110-x

34. A. Michels, J. de Boer, A. Bijl, Physica (Amsterdam) 4, 981 (1937). DOI 10.1016/S0031-8914(37)80196-2

35. V. Fock, Z. Phys. 47, 446 (1928). DOI 10.1007/BF01390750

36. C.G. Darwin, Math. Proc. Cambridge Philos. Soc. 27, 86 (1931). DOI 10.1017/S0305004100009373

37. R. Dutt, A. Mukherjee, Y.P. Varshni, Phys. Lett. A 280, 318 (2001). DOI 10.1016/S0375-9601(01)00067-6

38. J.F. da Silva, F.R. Silva, E. Drigo Filho, Int. J. Quantum Chem. 116, 497 (2016). DOI 10.1002/qua.25084

39. A. Flores-Riveros, A. Rodríguez-Contreras, Phys. Lett. A 372(40), 6175 (2008). DOI 10.1016/j.physleta.2008.08. 027 
40. M.F. Hasoglu, H.L. Zhou, S.T. Manson, Phys. Rev. A 93, 022512 (2016). DOI 10.1103/PhysRevA.93.022512

41. S.B. Doma, F.N. El-Gammal, J. Theor. Appl. Phys. 6, 28 (2012). DOI 10.1186/2251-7235-6-28

42. J.P. Connerade, Eur. Phys. J. D 74, 211 (2020). DOI 10.1140/epjd/e2020-10414-y

43. S. Saha, J. Jose, Int. J. Quantum Chem. 120(22), e26374 (2020). DOI 10.1002/qua.26374

44. M. Rodriguez-Bautista, R. Vargas, N. Aquino, J. Garza, Int. J. Quantum Chem. 118(13), e25571 (2018). DOI 10.1002/qua.25571

45. S. LumbTalwar, S. Lumb, V. Prasad, Eur. Phys. J. D 75, 59 (2021). DOI 10.1140/epjd/s10053-021-00064-0

46. A.M. Maniero, C.R. de Carvalho, F.V. Prudente, Ginette Jalbert, J. Phys. B: At., Mol. Opt. Phys. 53(18), 185001 (2020). DOI 10.1088/1361-6455/ab9f0f

47. H. de Oliveira Batael, E. Drigo Filho, J. Chahine, Eur. Phys. J. D 75, 52 (2021). DOI 10.1140/epjd/ s10053-021-00057-z

48. F.V. Prudente, M.N. Guimarães, in Electronic Structure of Quantum Confined Atoms and Molecules, ed. by K.D. Sen (Springer, Cham, 2014), chap. 5, pp. 101-143. DOI 10.1007/978-3-319-09982-8_5

49. R. Hernández-Esparza, B. Landeros-Rivera, R. Vargas, J. Garza, Ann. Phys. (Berlin) 531(7), 1800476 (2019). DOI 10.1002/andp. 201800476

50. L.F. Pasteka, T. Helgaker, T. Saue, D. Sundholm, H.J. Werner, M. Hasanbulli, J. Major, P. Schwerdtfeger, Mol. Phys. 118(19-20), e1730989 (2020). DOI 10.1080/00268976.2020.1730989

51. W.S. Nascimento, F.V. Prudente, Quim. Nova 39, 757 (2016). DOI 10.5935/0100-4042.20160045

52. S. Saha, J. Jose, Phys. Rev. A 102, 052824 (2020). DOI 10.1103/PhysRevA.102.052824

53. N.L. Guevara, R.P. Sagar, R.O. Esquivel, J. Chem. Phys. 122, 084101 (2005). DOI 10.1063/1.1848092

54. L.D. Site, Int. J. Quantum Chem. 115(19), 1396 (2014). DOI 10.1002/qua.24823

55. H.T. Peng, Y.K. Ho, Entropy 17, 1882 (2015). DOI 10.3390/e17041882

56. A. Mohajeri, M. Alipour, Chem. Phys. 360, 132 (2009). DOI 10.1016/j.chemphys.2009.04.016

57. R.P. Sagar, N.L. Guevara, J. Chem. Phys. 124, 134101 (2006). DOI 10.1063/1.2180777

58. R.O. Esquivelt, A.N. Tripathi, R.P. Sagar, V.H.J. Smith, J. Phys. B: At. Mol. Opt. Phys. 25, 2925 (1992). DOI 10.1088/0953-4075/25/13/003

59. A.N. Tripathi, R.P. Sagar, R.O. Esquivel, V.H.J. Smith, Phys. Rev. A 45, 4385 (1992). DOI 10.1103/PhysRevA.45. 4385

60. R.P. Sagar, M. Hô, J. Mex. Chem. Soc. 52(1), 60 (2008)

61. I. Bialynicki-Birula, J. Mycielski, Commun. Math. Phys. 44, 129 (1975). DOI 10.1007/BF01608825

62. H.H. Corzo, H.G. Laguna, R.P. Sagar, J. Math. Chem. 50, 233 (2012). DOI 10.1007/s10910-011-9908-2

63. E.A. Hylleraas, in Advances in Quantum Chemistry, vol. 1, ed. by P.O. Löwdin (Academic Press, 1964), pp. 1-33. DOI 10.1016/S0065-3276(08)60373-1

64. F.S. Carvalho, J.P. Braga, Quim. Nova 40(10), 1259 (2017). DOI 10.21577/0100-4042.20170125

65. C.A. Ten Seldam, R. De Groot, Physica 18(11), 891 (1952). DOI 10.1016/S0031-8914(52)80223-X

66. X.Y. Pan, V. Sahni, L. Massa, arXiv-physics/0310128 (2003)

67. B.M. Gimarc, J. Chem. Phys. 47(12), 5110 (1967). DOI 10.1063/1.1701767

68. S. Chandrasekhar, Astrophys. J. 100, 176 (1944). DOI 10.1086/144654

69. R.N. Hill, Phys. Rev. Lett. 38(12), 643 (1977). DOI 10.1103/PhysRevLett.38.643

70. H.A. Bethe, E.E. Salpeter, Quantum Mechanics of One- and Two-electron Atoms (Springer-Verlag, Berlin, 1977)

71. C. Le Sech, A. Banerjee, J. Phys. B: At. Mol. Opt. Phys. 44(10), 105003 (2011). DOI 10.1088/0953-4075/44/10/ 105003

72. C.N. Isonguyo, K.J. Oyewumi, O.S. Oyun, Int. J. Quantum Chem. 118(15), e25620 (2018). DOI 10.1002/qua.25620

73. N. Aquino, A. Flores-Riveros, J. Rivas-Silva, Phys. Lett. A 377(34), 2062 (2013). DOI 10.1016/j.physleta.2013.05. 048

74. F.G. F. A. de Saavedra, E. Buendía, Z Phys D - Atoms, Molecules and Clusters 38, 25 (1996). DOI 10.1007/ s004600050058

75. C.H. Lin, Y.K. Ho, Chem. Phys. Lett. 633, 261 (2015). DOI 10.1016/j.cplett.2015.05.029

76. R.G. Parr, Y. Weitao, Density-functional theory of atoms and molecules. International series of monographs on chemistry 16 (Oxford University Press, 1994)

77. Á. Nagy, Phys. Rep. 298(1), 1 (1998). DOI 10.1016/S0370-1573(97)00083-5

78. N.L. Guevara, R.P. Sagar, R.O. Esquivel, J. Chem. Phys. 119(14), 7030 (2003). DOI 10.1063/1.1605932

79. H. Montgomery, N. Aquino, A. Flores-Riveros, Phys. Lett. A 374(19), 2044 (2010). DOI 10.1016/j.physleta.2010. 02.074

80. N. Aquino, A. Flores-Riveros, J. Rivas-Silva, Phys. Lett. A 307(5), 326 (2003). DOI 10.1016/S0375-9601(02) 01767-X

81. A. Flores-Riveros, N. Aquino, H. Montgomery, Phys. Lett. A 374(10), 1246 (2010). DOI 10.1016/j.physleta.2009. 12.062

82. T.N. Barbosa, M.M. Almeida, F.V. Prudente, J. Phys. B: At., Mol. Opt. Phys. 48(5), 055002 (2015). DOI 10.1088/ 0953-4075/48/5/055002

83. F.V. Prudente, L.S. Costa, J.D.M. Vianna, J. Chem. Phys. 123(22), 224701 (2005). DOI 10.1063/1.2131068 